\title{
Temperature-induced dynamical conformational disorder in 4-vinyl benzoic acid molecular crystals: A molecular simulation study
}

\author{
N. Arul Murugan ${ }^{\text {a) }}$ \\ Solid State and Structural Chemistry Unit, Indian Institute of Science, Bangalore-560012, India
}

\begin{abstract}
Extensive molecular simulations are carried out as a function of temperature to understand and quantify the conformational disorder in molecular crystals of 4-vinyl benzoic acid. The conformational disorder is found to be dynamic and associated with a flip-flop motion of vinyl groups. The population of minor conformer is less than $3 \%$ up to $300 \mathrm{~K}$ and is $13.2 \%$ at $350 \mathrm{~K}$ and these results are consistent with the experimental observations. At still higher temperatures, the population of minor conformer increases up to $25 \%$. The evolution of structure at both molecular and unit-cell level of the molecular crystal as a function of temperature has been characterized by various quantities such as radial distribution functions, average cell parameters, volume, and interaction energies. The van't Hoff plot shows a nonlinear behavior at lower temperatures as it has been reported recently by Ogawa and co-workers in the case of stilbene, azobenzene [J. Am. Chem. Soc. 126, 3539 (2004)], and N-(4-methylbenzylidene)-4-methylaniline [Acta Crystallogr, Sect. B. Struct. Sci. B60, 589 (2004)] molecular crystals. A set of rigid body simulations were also carried out to quantify the effect of conformational disorder on structural quantities such as unit-cell volume and interaction energy. The anomalous shrinkage of vinyl $\mathrm{C}=\mathrm{C}$ bond length as a function of temperature has been explained by combining the results of simulations and a set of constrained optimizations using ab initio electronic structure calculations for various molecular structures differing in torsional angle.
\end{abstract}

\section{INTRODUCTION}

Experimental studies carried out by Mott ${ }^{1}$ and Anderson ${ }^{2}$ showed the importance of treating disorder as a fundamental concept in understanding the physical behavior of a condensed-phase system with considerable disorder. There

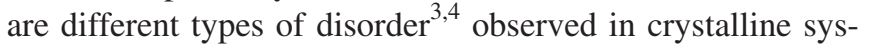
tems associated with various structural degrees of freedom of molecules. Globular-shape molecules such as adamantane ${ }^{5,6}$ and its substituted compounds ${ }^{7,8}$ are known to show disorder associated with orientational degrees of freedom resulting in the well-known plastic crystalline phase. ${ }^{8}$ In the case of crystals of 2-methyl-1,3-cyclohexane dione ${ }^{9}$ the disorder is associated with the onset of large-amplitude vibrations of methylene groups. In the case of molecular crystals of stilbene and azobenzene ${ }^{10-13}$ the disorder results in a minor conformer, which is related to the major conformer by a twofold rotation about the long molecular axis.

Recent $\mathrm{x}$-ray-diffraction studies ${ }^{14}$ on 4 -vinyl benzoic acid at high temperatures report a conformational disorder associated with the flip-flop motion of vinyl groups (see Fig. 1). The $-\mathrm{COOH}$ groups (connected to carbon labeled as $\mathrm{C} 1$ in Fig. 2) are not associated with any conformational disorder as these groups (of different molecules) are bonded through hydrogen bonding.

4-vinyl benzoic acid crystallizes in $P 2_{1} / n$ space group with $Z=4$ at $108 \mathrm{~K}^{15}$ At low temperatures the vinyl group $\left(-\mathrm{CH}=\mathrm{CH}_{2}\right)$ is almost coplanar with the phenyl ring and

${ }^{a)}$ Electronic mail: murugan@sscu.iisc.ernet.in the molecules are described by a torsional angle $\phi$ (between phenyl and vinyl groups) closer to $0^{\circ}$. At higher temperatures, the vinyl group is no longer coplanar with the phenyl ring and a large-amplitude torsional motion of vinyl groups results in a minor conformer which is described by a torsional angle closer to $180^{\circ}$. Throughout this manuscript, the torsional angle $\phi$ refers to the angle between the phenyl and vinyl groups. The population of minor conformer has been quantified to be less than $3 \%$ up to $300 \mathrm{~K}$ based on crystallographic structure elucidation with a disorder model at various temperatures. The population of minor conformer increases by $11.8 \%$ at $363 \mathrm{~K} .{ }^{14}$

It has been observed by Yasuda et al. ${ }^{14}$ that the vinyl $\mathrm{C}=\mathrm{C}$ bond length shows an anomalous decrease as a function of temperature. The bond lengths are 1.325, 1.288, and $1.276 \AA$ at temperatures 108,293 , and $363 \mathrm{~K}$, respectively. ${ }^{14}$ This variation has been partly attributed to the disorder associated with the torsional vibration of the vinyl group in 4-vinyl benzoic acid.

In the present study, we have carried out isothermalisobaric variable shape simulations for the 4-vinyl benzoic acid molecular crystal as a function of temperature. To quantify the disorder associated with the torsional vibration of the vinyl group, the torsional mode has been included explicitly in the simulations.

The disordered proton in the carboxylic acid dimer group is not included explicitly in these calculations for the following reasons: (i) The interatomic distances between the disordered proton and the carbons of the vinyl groups are considerably larger (i.e., 4.34, 4.43, 4.74, and $5.18 \AA$ ), so the 

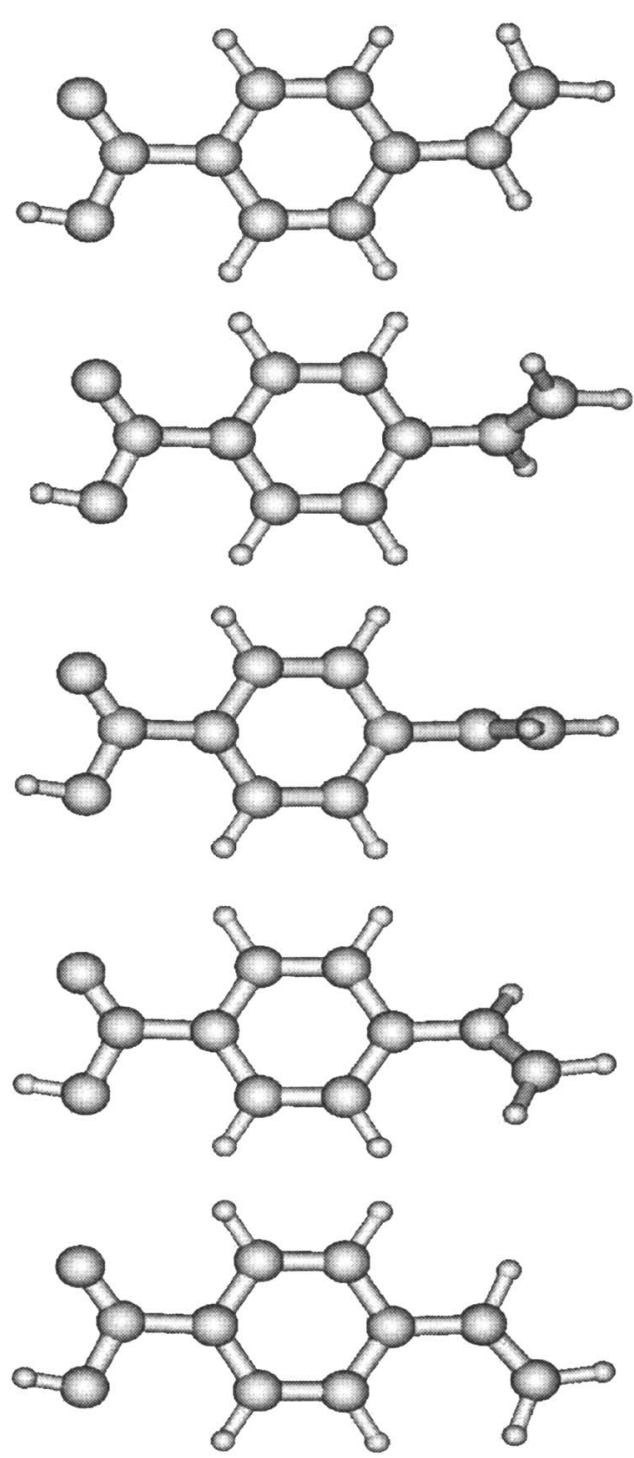

FIG. 1. Flip-flop motion of vinyl group in 4-vinyl benzoic acid.

effect of disordered proton on the barrier associated with the flip-flop motion of the vinyl groups may not be very significant. (ii) In the literature, we are not aware of any force field to include the disordered proton in organic molecular crystals in the simulations. (iii) The proton is considerably lighter in mass than the vinyl groups, so the dynamics of the proton is considerably faster than the dynamics of the vinyl groups. Hence, the vinyl groups may actually see the average position of the disordered hydrogens and, in that case, the results may not be very different from what we have presented in

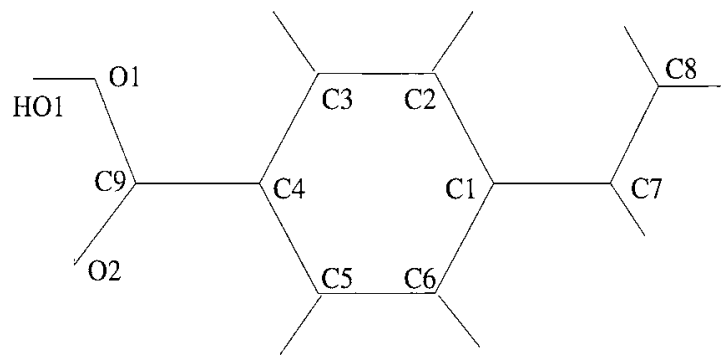

FIG. 2. Molecular structure of 4-vinyl benzoic acid with labeling. the manuscript. (iv) As the proton is lighter, we may have to adapt more accurate but highly expensive Car-Parrinello molecular-dynamics ${ }^{16}$ (CPMD) method. This method will provide a quantitative estimate of the contribution of the carboxylic proton to the barrier associated with the flipping of vinyl groups. But we are interested in investigating the structure of 4-vinyl benzoic acid as a function of temperature with a simpler potential model.

The intramolecular potential function to describe the torsional vibration of the vinyl groups has been derived from $a b$ initio electronic structure calculations, using GAUSSIAN98 computational package. ${ }^{17}$ The structure of the crystal as a function of temperature is characterized with various structural quantities such as average cell parameters, volume, energy, and radial distribution functions (rdfs). van't Hoff plot has been calculated from the temperature variation of the relative population of major and minor conformers. The activation energy for the flip-flop motion of vinyl groups has been estimated from the number of jumps across the barrier associated with this motion. By carrying out constrained structural optimization using the $a b$ initio electronic structure calculation software, for 4-vinyl benzoic acid with varying torsional angle $\phi$ and using the temperature dependence data of torsional angle distributions from simulations, the temperature dependence of vinyl $\mathrm{C}=\mathrm{C}$ and $\mathrm{C}-\mathrm{Ph}$ bond lengths has been calculated.

\section{METHODS}

\section{A. Intermolecular potential}

The intermolecular potential function is Buckingham 6-exp type without charge as given in Eq. (1). The potential parameters used are those derived by Filippini and Gavezzotti (FG). ${ }^{18}$ These potential parameters are based on the distribution of atom-atom distances in 1846 organic crystals, structural data of 217 organic crystals, and the heats of sublimation of 122 compounds. Although no electrostatic interaction is included explicitly in the FG potential, Filippini and Gavezzotti ${ }^{18}$ suggest that it is included implicitly by the use of effective potential parameters which lead to an additional lowering of the well depth of the (6-exp) potential for cross or unlike interactions. In the present calculations, the well depth of the potential function is scaled by 0.8 to get a better agreement between the melting point calculated from simulations and the experimental melting point. This is achieved by multiplying the potential parameters $A_{\mu \nu}$ and $B_{\mu \nu}$ by 0.8 . The experimentally observed melting point is $416 \mathrm{~K}$ (Ref. 15) and in the present calculations, 4-vinyl benzoic acid melts between the temperature range 390-400 K. The potential parameters used in our simulations are listed in Table I.

It is not very uncommon to observe higher melting temperatures than experimentally calculated melting temperatures for the simulated molecular crystals. This deviation of melting temperatures calculated from simulations has been attributed to the superheating effect. ${ }^{19,20}$ Velardez et al. ${ }^{21}$ Sprik et al., ${ }^{22}$ and Greig and Pawley ${ }^{23}$ report relatively higher transition temperatures in simulated solid poly(tetrafluroethylene), ${ }^{21}$ ammonium dinitramide, ${ }^{22}$ and adamantane, respectively. ${ }^{23}$ The better agreement between 
TABLE I. Potential parameters for 4-vinyl benzoic acid.

\begin{tabular}{cccc}
\hline \hline & FG potential & & \\
Type & $A$ & $B$ & $C$ \\
O-O & $\left(\mathrm{kJ} / \mathrm{mol}^{6}\right)$ & $(\mathrm{kJ} / \mathrm{mol})$ & $\left(\AA^{-1}\right)$ \\
$\mathrm{O}-\mathrm{C}$ & 1067.76 & 156247.30 & 3.74 \\
$\mathrm{O}-\mathrm{H}$ & 2145.56 & 314469.44 & 3.74 \\
$\mathrm{C}-\mathrm{C}$ & 351.46 & 236345.80 & 4.82 \\
$\mathrm{C}-\mathrm{H}$ & 1934.68 & 180916.16 & 3.47 \\
$\mathrm{H}-\mathrm{H}$ & 378.23 & 96633.67 & 4.10 \\
& 87.36 & 19326.73 & 4.01 \\
$c_{0}$ & & & \\
$(\mathrm{~kJ} / \mathrm{mol})$ & $c_{1}$ & $c_{2}$ & $c_{3}$ \\
0.017 69 & $(\mathrm{kJ} / \mathrm{mol})$ & $(\mathrm{kJ} / \mathrm{mol})$ & $(\mathrm{kJ} / \mathrm{mol})$ \\
\hline \hline
\end{tabular}

experimental and simulated transition temperatures was obtained when the potential was scaled or when the defects were introduced $^{22}$ within the molecular crystals. Here, in the present calculations we have scaled the potential instead of introducing defects. This is because of the reason that it was necessary to start with a completely ordered system to understand whether the nature of the experimentally observed conformational disorder is static or dynamic.

$$
u\left(r_{i j, \mu \nu}\right)=-\frac{A_{\mu \nu}}{\left(r_{i j, \mu \nu}\right)^{6}}+B_{\mu \nu} \exp \left(-C_{\mu \nu} r_{i j, \mu \nu}\right) .
$$

Here $i, j$ refer to molecular indices and $\mu, \nu$ to atomic indices.

\section{B. Intramolecular potential}

To our knowledge, the intramolecular potential function to model the torsional vibration of the vinyl group was not available in the literature. So, it has been derived by densityfunctional (DFT) calculations [implemented in the software GAUSSIAN98 (Ref. 17)] using Becke three-parameter method and Lee-Yang-Parr electron correlation functional with 6$31 \mathrm{G}(d)$ basis set. This involves a set of constrained optimizations where the torsional angle $\phi$ has been constrained to various angles from $0^{\circ}$ to $180^{\circ}$ in the increment of $10^{\circ}$. The $a b$ initio energies obtained as a function of torsional angle are fitted to a function of the form in the Eq. (2). Figure 3 shows a fit for the $a b$ initio energies as a function of torsional angle. The values of the coefficients are scaled by a factor of 0.4 and the final values are listed in Table I. The scaling has been done to observe the flip-flop motion of vinyl groups at the correct temperature as it has been reported experimentally. Also, it has been reported that $a b$ initio electronic structure calculations always do not correctly reproduce the rotational barriers even though the form of the potential-energy function associated with the intramolecular motion is reproduced sufficiently well. ${ }^{24}$ This scaling factor is justified on the basis of better agreement of many quantities (associated with the flip-flop motion of vinyl groups) with corresponding experimentally calculated quantities. The intramolecular energy for the $i$ th molecule is given by

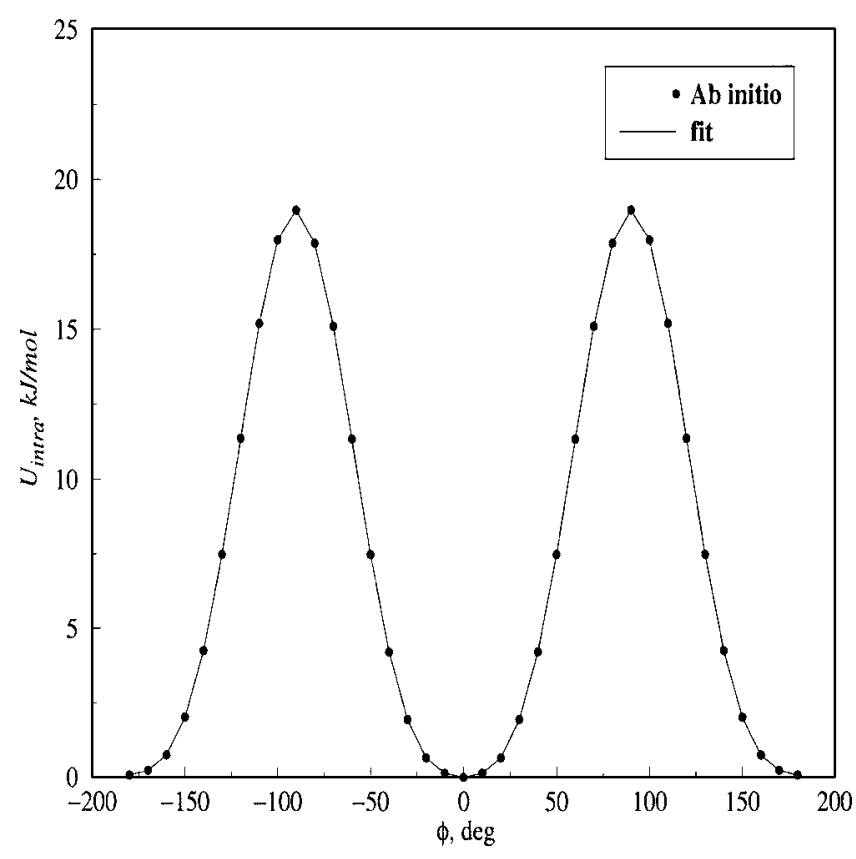

FIG. 3. Intramolecular potential function derived from $a b$ initio electronic structure calculations to include the torsional motion of vinyl group in simulations.

$$
\begin{aligned}
u_{\text {intra }}\left(\phi_{i}\right)= & c_{0}+c_{1}\left(1.0-\cos \left(2 \phi_{i}\right)\right)+c_{2}\left(1.0-\cos \left(4 \phi_{i}\right)\right) \\
& +c_{3}\left(1.0-\cos \left(6 \phi_{i}\right)\right)
\end{aligned}
$$

where $\phi_{i}$ is the torsional angle in radian for the $i$ th molecule.

\section{Variable shape Monte Carlo simulations}

Simulations have been carried out in isothermal-isobaric or NPT ensemble with variable shape simulation ${ }^{25}$ cell using the Monte Carlo method and the importance sampling algorithm of Metropolis. ${ }^{26}$ The simulation cell is represented by a
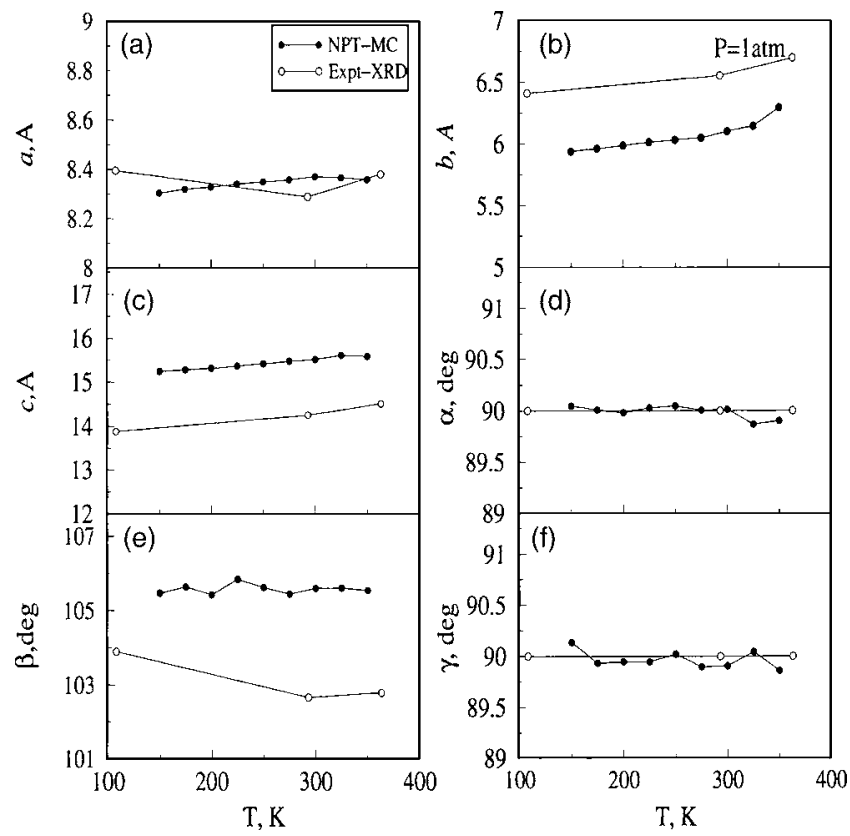

FIG. 4. Temperature dependence of cell parameters compared with experimentally calculated cell parameters (a) $a$, (b) $b$, (c) $c$, (d) $\alpha$, (e) $\beta$, and (f) $\gamma$. 


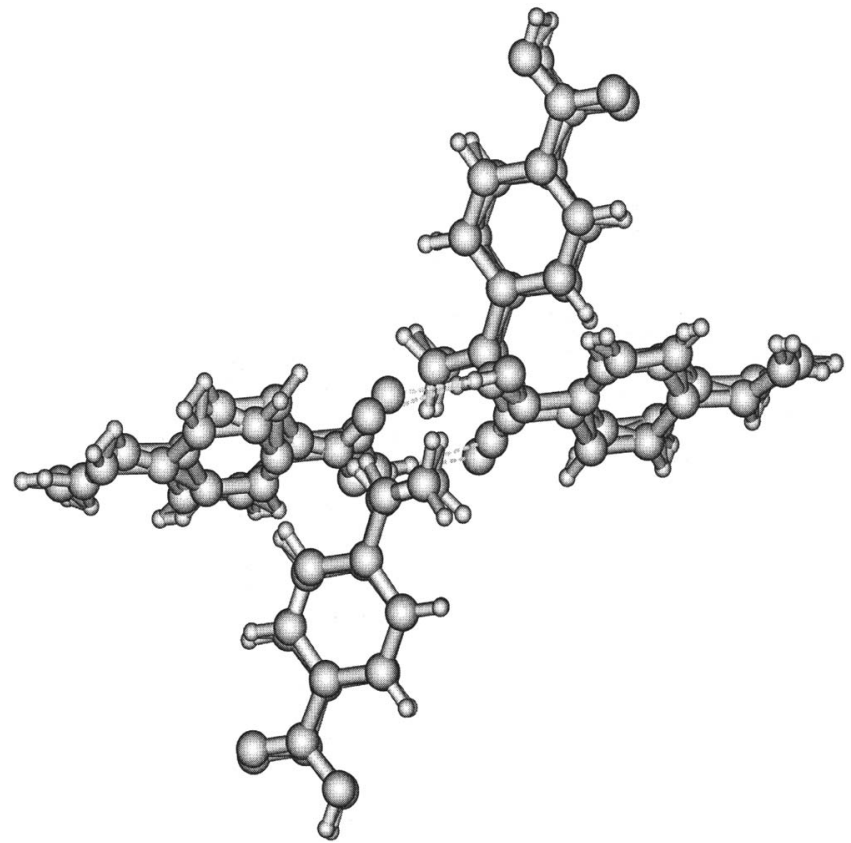

FIG. 5. A 3D overlay of the molecules within a unit cell obtained from simulation at $108 \mathrm{~K}$ and $1 \mathrm{~atm}$ pressure with that of experiment (at $108 \mathrm{~K}$ and $1 \mathrm{~atm}$ pressure).

minimum of six degrees of freedom after the subsequent modification by Yashonath and Rao, ${ }^{27}$ while the original formulation by Parrinello and Rahman ${ }^{25}$ used nine degrees of freedom to explain simulation cell. There are two sets of calculations: one incorporating flexibility for molecules through including the torsional mode and another with a rigid-body assumption for molecules. In the case of flexiblebody (FB) calculations, each molecule has seven degrees of

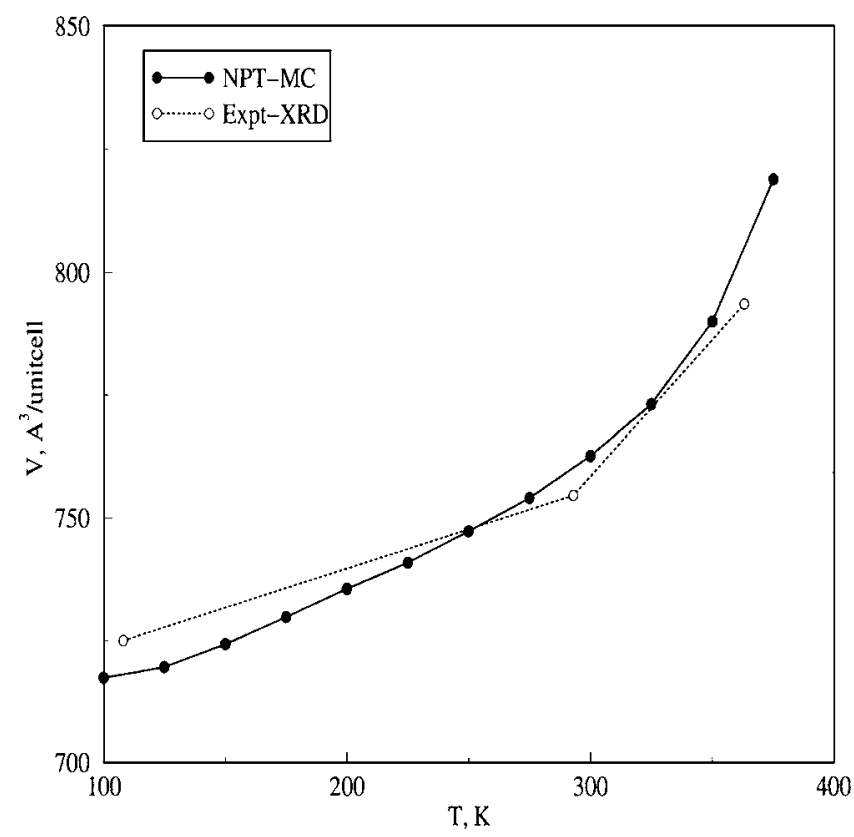

FIG. 6. Temperature dependence of unit-cell volume as a function of temperature compared with experimental unit-cell volume.

freedom including three for translation, three for rotation, and one to include the torsional/flip-flop motion of the vinyl group. Thus, the average of any property $a$ is obtained by integrating over these $(7 N+6)$ variables as in Eq. (3). In the case of rigid-body (RB) calculations, each molecule has six degrees of freedom including three for translational and three for rotational motion and the average of any property $a$ is obtained by integrating over these $(6 N+6)$ variables as in Eq. (4).

$$
\begin{aligned}
& \langle a\rangle=\frac{\int d \mathbf{a} \int d \mathbf{b} \int d \mathbf{c} \int d \mathbf{\Omega}^{N} \int d \phi^{N} \int d \mathbf{r}^{N} a\left(\mathbf{r}^{N}, \mathbf{\Omega}^{N}, \phi^{N}\right) p\left(\mathbf{r}^{N}, \mathbf{\Omega}^{N}, \phi^{N}\right)}{\int d \mathbf{a} \int d \mathbf{b} \int d \mathbf{c} \int d \mathbf{\Omega}^{N} \int d \phi^{N} \int d \mathbf{r}^{N} p\left(\mathbf{r}^{N}, \mathbf{\Omega}^{N}, \boldsymbol{\phi}^{N}\right)}, \\
& \langle a\rangle=\frac{\int d \mathbf{a} \int d \mathbf{b} \int d \mathbf{c} \int d \mathbf{\Omega}^{N} \int d \mathbf{r}^{N} a\left(\mathbf{r}^{N}, \boldsymbol{\Omega}^{N}\right) p\left(\mathbf{r}^{N}, \mathbf{\Omega}^{N}\right)}{\int d \mathbf{a} \int d \mathbf{b} \int d \mathbf{c} \int d \mathbf{\Omega}^{N} \int d \mathbf{r}^{N} p\left(\mathbf{r}^{N}, \mathbf{\Omega}^{N}\right)}
\end{aligned}
$$

where $N$ refers to the number of molecules in the simulation box and $p\left(\mathbf{r}^{N}, \boldsymbol{\Omega}^{N}, \phi^{N}\right)=e^{-\beta U}\left(\mathbf{r}^{N}, \boldsymbol{\Omega}^{N}, \phi^{N}\right)$ and $a\left(\mathbf{r}^{N}, \boldsymbol{\Omega}^{N}, \phi^{N}\right)$ are the probability and the property, respectively, for each configuration specified by $\left(\mathbf{r}^{N}, \boldsymbol{\Omega}^{N}, \phi^{N}\right)$ in the case of FB calculations. Similarly, $p\left(\mathbf{r}^{N}, \mathbf{\Omega}^{N}\right)=e^{-\beta U}\left(\mathbf{r}^{N}, \boldsymbol{\Omega}^{N}\right)$ and $a\left(\mathbf{r}^{N}, \mathbf{\Omega}^{N}\right)$ are the probability and the property, respectively, for each configuration specified by $\left(\mathbf{r}^{N}, \boldsymbol{\Omega}^{N}\right)$ in the case of RB calculations. Here $\mathbf{r}^{N}$ are the center-of-mass positions of $N$ molecules and $\boldsymbol{\Omega}^{N}$ specifies the orientations of $N$ molecules as a whole, while $\phi^{N}$ specifies the torsional angles of the $N$ molecular species. Note that $\mathbf{r}$ and $\boldsymbol{\Omega}$ have three components each: $\mathbf{r}$ specifies the three Cartesian coordinates and $\boldsymbol{\Omega}$ the three Euler angles.

\section{Computational details}

The simulation box contains $4 \times 5 \times 3$ unit cells (space group $=P 2_{1} / n$ and $Z=4$ ) and a total of 240 molecules. Two sets of simulations were carried out with flexible-body and rigid-body models for molecules. (i) In the case of flexiblebody calculations, the simulations were carried out at $1 \mathrm{~atm}$ pressure and the temperatures starting with 100 up to $375 \mathrm{~K}$ in the increment of $25 \mathrm{~K}$ and also at temperatures 390 and $400 \mathrm{~K}$. For the calculation at $100 \mathrm{~K}$ and $1 \mathrm{~atm}$ the initial structure is taken from the crystallographic data provided by Yasuda et al. ${ }^{15}$ For calculations at other temperatures, the initial configuration is taken from the final configuration of the previous low-temperature calculation. Each Monte Carlo 
move (MCM) of a molecule consists of a random translation of the center of mass (c.o.m.) of the molecule, a random rotation of the molecule by a small angle along the randomly selected axis, and a random torsional rotation along the $\mathrm{C}-\mathrm{Ph}$ bond of the molecule. Each MCM pertaining to the simulation cell consists of a random displacement of all the cell parameters by a small value. Each Monte Carlo step (MCS) involves MCMs of all $N$ molecules once and MCM of the simulation box once. The maxima associated with the translational, rotational, and torsional displacements of molecules and the displacement of cell vectors are adjusted so that the acceptance ratio of MCMs is around 0.40. Each run involves $18000 \mathrm{MCSs}$, including $3000 \mathrm{MCSs}\left(7.2 \times 10^{5} \mathrm{MCMs}\right)$ for equilibration. The calculation of various structural quantities such as rdfs, average cell parameters, volume, and various interaction energies are averaged over 15000 MCSs (3.6 $\left.\times 10^{6} \mathrm{MCMs}\right)$ after equilibration. It has been assured that the system has equilibrated in all these calculations and the structural quantities are calculated over a reasonably longenough ensemble average. The c.o.m.-c.o.m. cutoff of $13 \AA$ has been considered for the calculation of interaction energies. (ii) The rigid-body calculations are carried out at temperatures of 100, 150, 200, 250, 300, and $375 \mathrm{~K}$ and at $1 \mathrm{~atm}$ pressure. The initial configuration for the calculation at $100 \mathrm{~K}$ and $1 \mathrm{~atm}$ is taken again from the crystallographic structure given by Yasuda et al. ${ }^{15}$ For all other calculations the initial configuration is taken from the final configuration of previous low-temperature calculations. In the case of RB calculations, each MCM of a molecule consists only of a random translation of the c.o.m. of the molecule and a random rotation of the molecule by a small angle along a randomly selected axis. The structural quantities are calculated as an average over 15000 MCSs after equilibration over 3000 MCSs.

\section{E. Ab initio calculations}

The DFT calculations using Becke three-parameter method and Lee-Yang-Parr electron correlation functional with 6-31G $(d)$ basis set are carried out to get the optimized structure of a single molecule of 4-vinyl benzoic acid for various torsional angles, $\phi$. The constrained optimizations of the molecule involve freezing of a single torsional degree of freedom $(\phi)$ and all other degrees of freedom are allowed to vary. This set of calculations provide the variation of all structural parameters such as bond lengths, angles, and torsional angles as a function of torsional angle $\phi$.

\section{RESULTS AND DISCUSSIONS}

\section{A. Structure and energetics}

Figure 4 shows the variation of cell parameters $a, b, c$, $\alpha, \beta$, and $\gamma$ as a function of temperature along with the experimentally reported cell parameters by Yasuda et al. ${ }^{14,15}$ The cell parameters $a, \alpha, \beta$, and $\gamma$ are reproduced well within $1 \%$ compared to experimentally reported quantities. The cell parameter $b$ is $8 \%$ higher than the experimentally reported $b$, while $c$ is $3 \%$ less than the experimentally reported value. Figure 5 shows a 3D overlay of the molecules within a unit cell obtained from simulations (at $108 \mathrm{~K}$ and
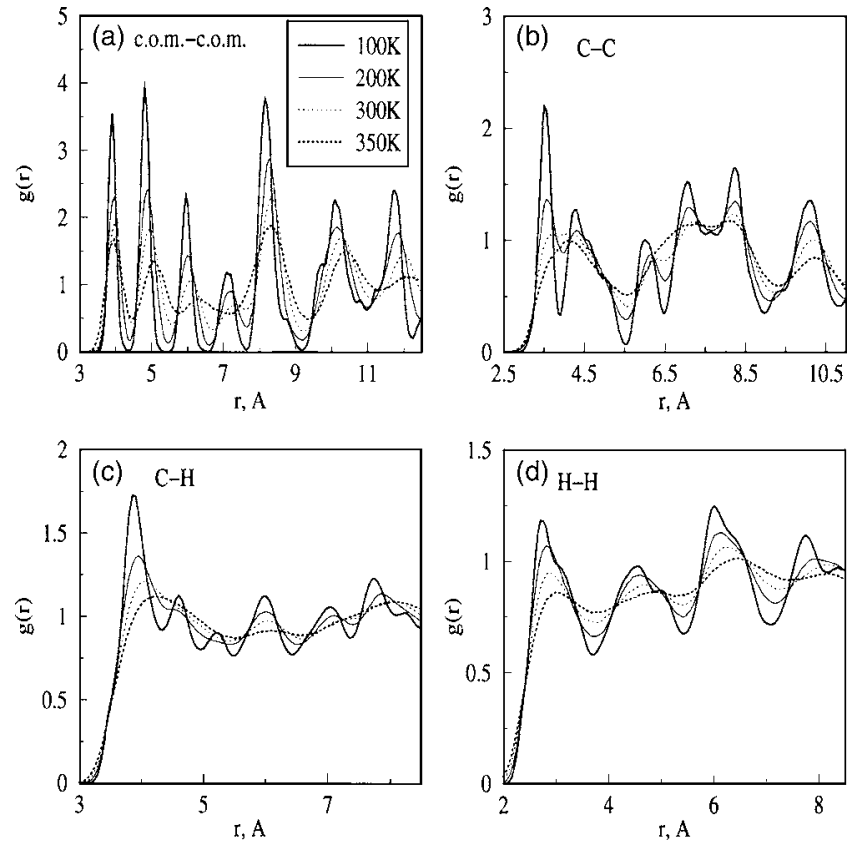

FIG. 7. Variation of c.o.m.-c.o.m. and atom-atom rdfs as a function of temperature. (a) c.o.m.-c.o.m., (b) C-C, (c) C-H, and (d) H-H.

$1 \mathrm{~atm}$ ), with the experimental structure at $108 \mathrm{~K}$ and $1 \mathrm{~atm}$ pressure. It is clearly seen from this figure that there is a better agreement between the simulated structure and the experimental crystallographic structure. It also shows that closer contacts are almost similar in both the structures. The slight difference between these two structures aries due to the fact that the simulated structure considered here for comparison is an instantaneous structure during a simulation and not an average structure. The difference between the experimental and simulated structures is also associated with the difference in the predicted cell parameters compared to the experimental cell parameters. Figure 6 shows the temperature dependence of the unit-cell volume compared to the experimentally calculated unit-cell volume. ${ }^{14,15}$ At all temperatures, the deviation between the experimental unit-cell volume and the simulated unit-cell volume is less than $2 \%$. Altogether, the potential parameters seem to reproduce the structure of the 4-vinyl benzoic acid crystals in the temperature range of $100-375 \mathrm{~K}$ reasonably well.

Figure 7 shows the variation of c.o.m.-c.o.m. rdf and $\mathrm{C}-\mathrm{C}, \mathrm{C}-\mathrm{H}$, and $\mathrm{H}-\mathrm{H}$ rdfs as a function of temperature. The well-defined peaks in the c.o.m.-c.o.m. rdf up to $350 \mathrm{~K}$ confirms the solid nature of the 4-vinyl benzoic acid molecular crystal at least up to this temperature. At $200 \mathrm{~K}$, one can see larger changes occurring in $\mathrm{C}-\mathrm{H}$ and $\mathrm{H}-\mathrm{H}$ rdfs and we will demonstrate later in this manuscript that it has its origin in the onset of conformational or intramolecular orientational disorder. Above $300 \mathrm{~K}$, the disappearance of the distinct peaks in various atom-atom rdfs suggest considerable increase in disorder within the system. This type of evolution of rdfs as a function of temperature has been observed in the case of other flexible systems such as stilbene, ${ }^{28}$ biphenyl, ${ }^{29}$ $p$-terpheny $\mathrm{l}^{30}$ having conformational disorder, and adamantane $\mathrm{e}^{31,32}$ and its substituted compounds having orientational disorder. The liquidlike feature appearing in the 


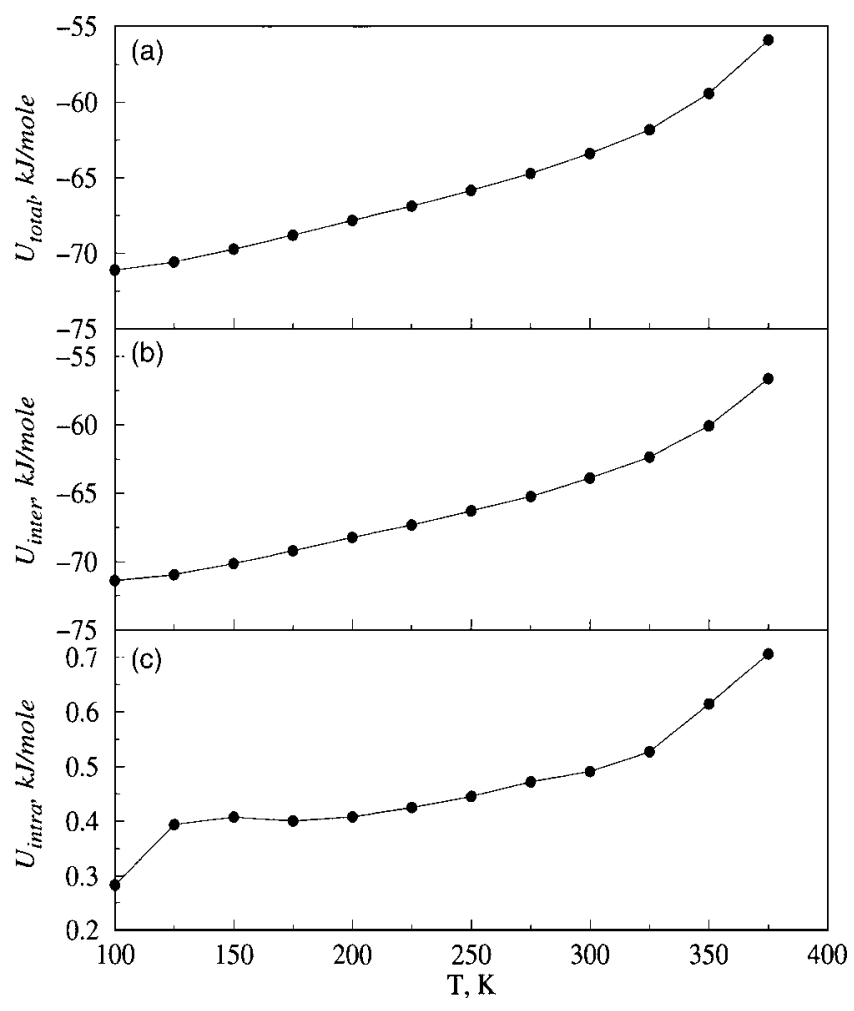

FIG. 8. Temperature dependence of various energies as a function of temperature at 1 atm pressure. (a) Total interaction energy, (b) intermolecular energy, and (c) intramolecular energy.

atom-atom rdfs is largely due to a partial or complete melting of torsional (as in the case of stilbene ${ }^{28}$ ) or rotational degrees of freedom (as in the case of globular-shape molecules). $8,31,33,34$ The liquidlike nature of various atomatom rdfs at higher temperatures in the present case is largely due to the partial melting of the torsional degrees of freedom, $\phi$, and to some extent due to the rotational and translational motion of molecules.

Figure 8 shows the variation of the total interaction energy $\left(U_{\text {total }}\right)$, intermolecular energy $\left(U_{\text {inter }}\right)$, and intramolecular energy $\left(U_{\text {intra }}\right)$ as a function of temperature. $U_{\text {inter }}, U_{\text {intra }}$, and $U_{\text {total }}$ are calculated from Eqs. (5)-(7), respectively. It is evident that the contribution of intramolecular energy to the total interaction energy is less than $1 \%$ :

$$
\begin{aligned}
& U_{\text {inter }}=\frac{1}{2 N} \sum_{i=1}^{N} \sum_{j=1}^{N} u_{i j}, \\
& U_{\text {intra }}=\sum_{i=1}^{N} u_{\text {intra }}\left(\phi_{i}\right), \\
& U_{\text {total }}=U_{\text {inter }}+U_{\text {intra }},
\end{aligned}
$$

where $N$ stands for the total number of molecules in the simulation box. The indices $i$ and $j$ run over all the molecules. The total intermolecular energy increases by $14.50 \mathrm{~kJ} / \mathrm{mol}$ for a temperature change from 100 to $375 \mathrm{~K}$, while the total intramolecular energy increases only by $0.42 \mathrm{~kJ} / \mathrm{mol}$. Energies and unit-cell volume show a monotonic increase as a function of temperature, suggesting that

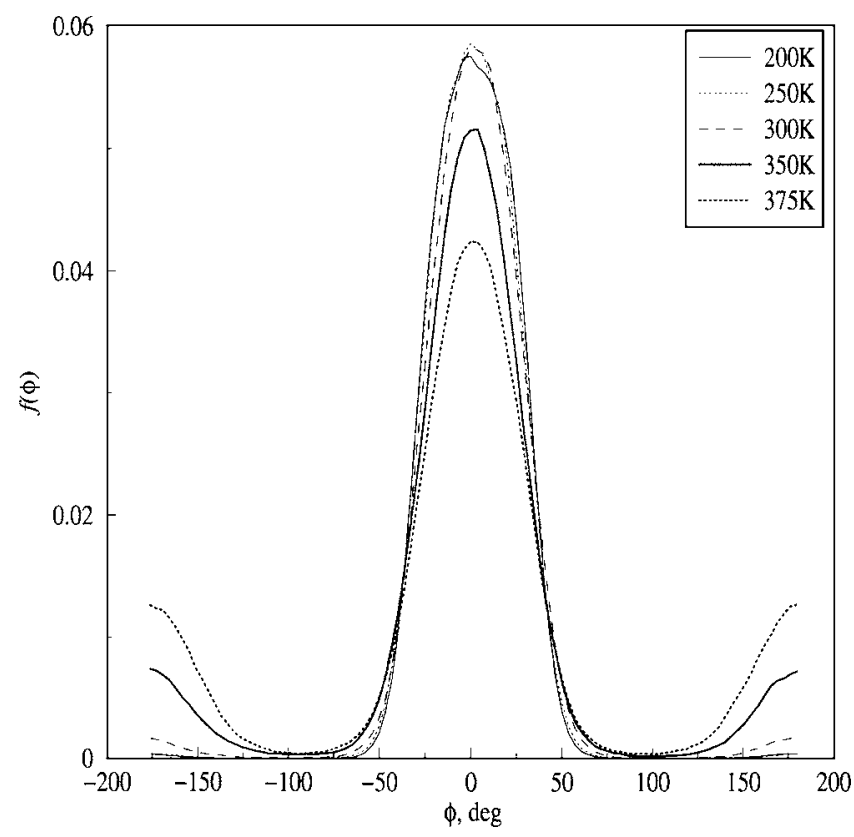

FIG. 9. The torsional angle distribution at different temperatures and at 1 atm pressure.

the partial melting of the torsional degrees of freedom $(\phi)$ is continuous and is not associated with any first-order phase transition.

\section{B. Nature of disorder}

Figure 9 shows the temperature dependence of the torsional angle distribution at different temperatures. At low temperatures up to $200 \mathrm{~K}$, the torsional angle of all the molecules is distributed between the range of angles $-60^{\circ}-60^{\circ}$. At moderate temperatures (up to $300 \mathrm{~K}$ ), it is evident from the figure that the torsional angle distribution extends over the whole range between $-180^{\circ}$ and $180^{\circ}$. At still higher temperatures (at and above $350 \mathrm{~K}$ ), one can see a considerable increase in the population of conformers with $|\phi|$ $\approx 180^{\circ}$. To quantify the disorder in the system, we have defined the molecules with $|\phi| \leqslant 90^{\circ}$ as major conformer and molecules with $|\phi|>90^{\circ}$ as minor conformer. Figure 10 shows the variation of percentage of minor conformers as a function of temperature. From this figure it is evident that the onset of conformational disorder occurs at $225 \mathrm{~K}$. Until $300 \mathrm{~K}$ the population of minor conformer is less than $3 \%$. This is consistent with crystallographic structure refinement (with a disorder model) results at $293 \mathrm{~K}$ in which the population of the minor conformer is reported to be less than $3 \%{ }^{14}$ At higher temperatures, the population of minor conformers steeply increases. At $350 \mathrm{~K}$, the population of minor conformers increases to $13.17 \%$, which can be compared to the experimentally calculated $11.8 \%$ at $363 \mathrm{~K} .{ }^{14}$ At $375 \mathrm{~K}$, the population of minor conformers is close to $25 \%$ and every one molecule in four is a minor conformer.

Figure 11 shows the potential-energy distribution as a function of torsional angle for various temperatures. At low temperatures up to $200 \mathrm{~K}$, the potential-energy distribution extends only over a range of torsional angles between $-60^{\circ}$ and $60^{\circ}$, the molecules exhibit a small-amplitude motion, 


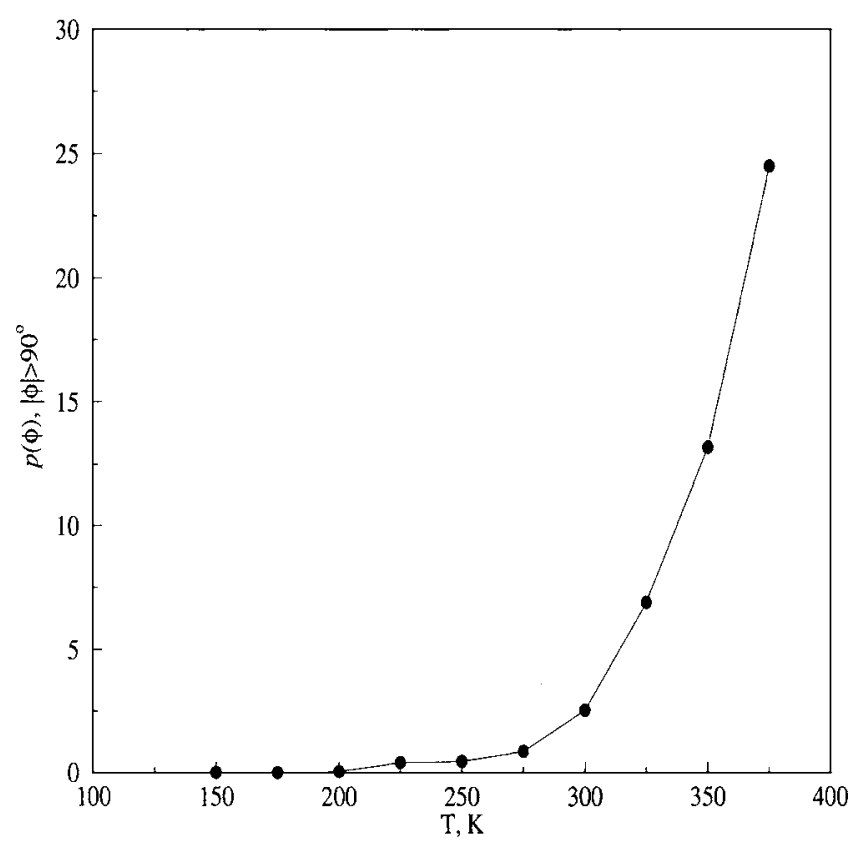

FIG. 10. The population of minor conformers as a function of temperatures. Minor conformers are defined as the molecules with $|\phi|>90^{\circ}$.

and the population of minor conformers remains zero. But at higher temperatures, the energy distribution extends over the whole range of torsional angles, from $-180^{\circ}$ to $180^{\circ}$ indicating a large-amplitude motion of at least a small fraction of molecules. It is clearly evident that the flip motion has a barrier near $\pm 90^{\circ}$. It is also evident that barriers at $+90^{\circ}$ and $-90^{\circ}$ are almost same and there may not be any preference of direction for flip motion of the vinyl groups. The barrier heights as functions of temperature are listed in Table II and shows a maximum at $300 \mathrm{~K}$. One interesting observation is that the minor and major conformers have almost the same

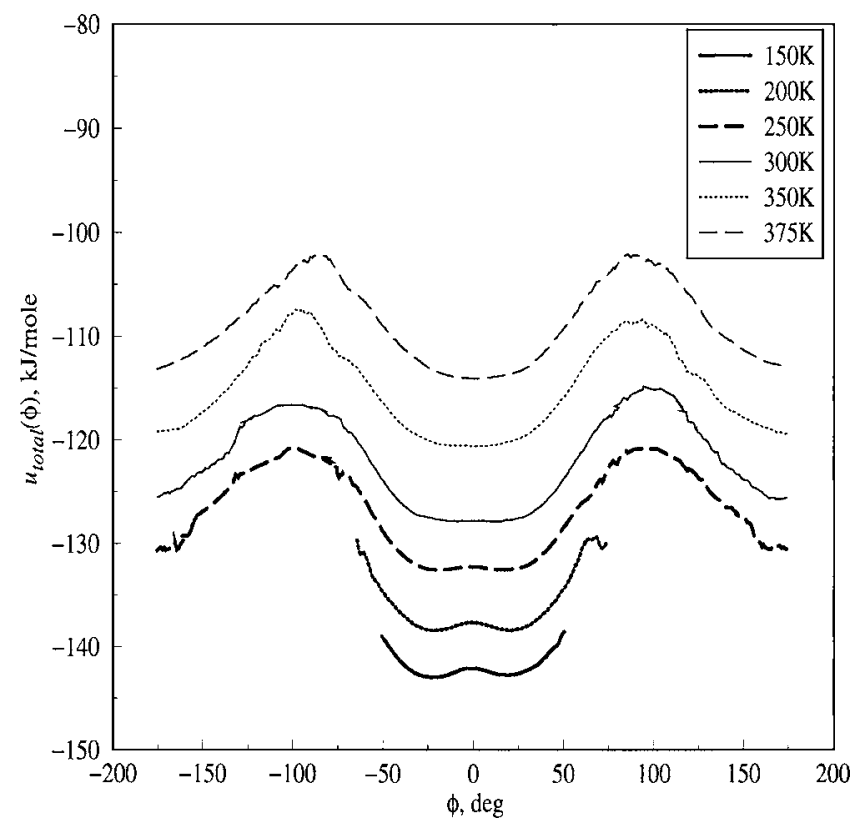

FIG. 11. Temperature dependence of potential-energy distribution as a function of torsional angle at $1 \mathrm{~atm}$ pressure. The distribution shows a barrier at $\pm 90^{\circ}$ for the flip motion.
TABLE II. The barrier heights for the flip motion as a function of temperature.

\begin{tabular}{cc}
\hline \hline $\begin{array}{c}\text { Temperature } \\
(\mathrm{K})\end{array}$ & $\begin{array}{c}\text { Barrier height } \\
(\mathrm{kJ} / \mathrm{mol})\end{array}$ \\
\hline 250.0 & 10.873 \\
300.0 & 11.203 \\
350.0 & 12.850 \\
375.0 & 11.861 \\
\hline
\end{tabular}

interaction energy. This is quite different from our earlier observation $^{28}$ in the case of stilbene molecular crystals, where the major and minor conformers have different interaction energies. This can be explained on the basis of molecular structure of both these molecules. In the case of stilbene, the interconversion to minor conformer requires a large spatial readjustment of neighboring molecules. But in the case of 4-vinyl benzoic acid the interconversion to minor conformer involves the torsional rotation of a small vinyl segment and, hence, neighboring molecular environment is not necessarily modified very much to accommodate the minor conformer. This can be a reason for the minor conformer having the same interaction energy as the major conformer in the case of 4-vinyl benzoic acid.

Figure 12 shows snapshots of crystal structure at tem-

(a)

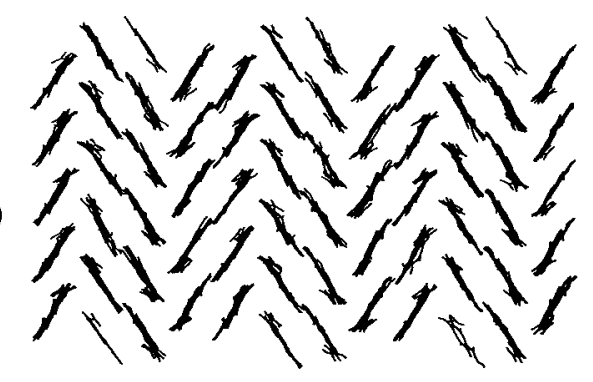

(b)
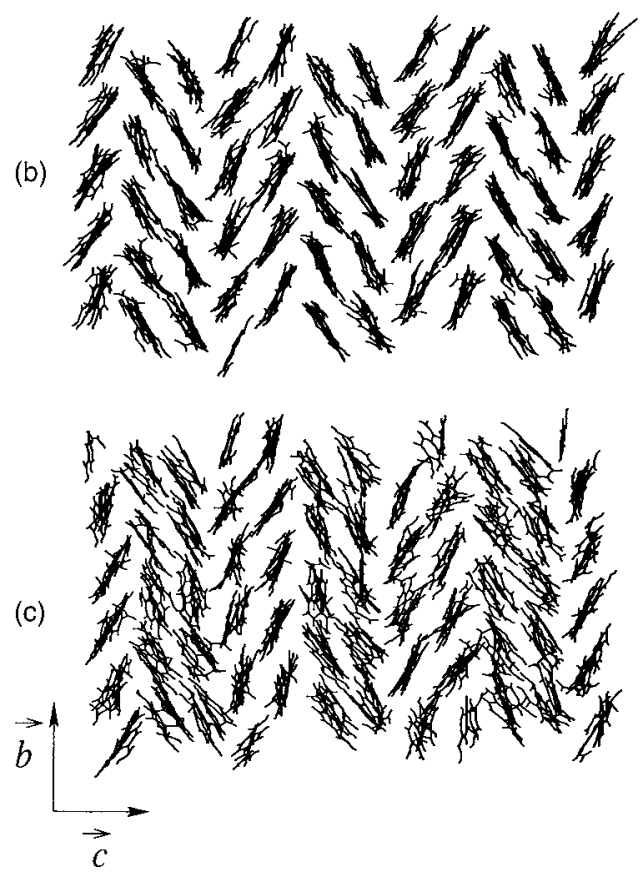

FIG. 12. Snapshots of crystal structure at (a) 100, (b) 350 , and (c) $375 \mathrm{~K}$ and at $1 \mathrm{~atm}$ pressure looking down the crystallographic $a$ axis. 


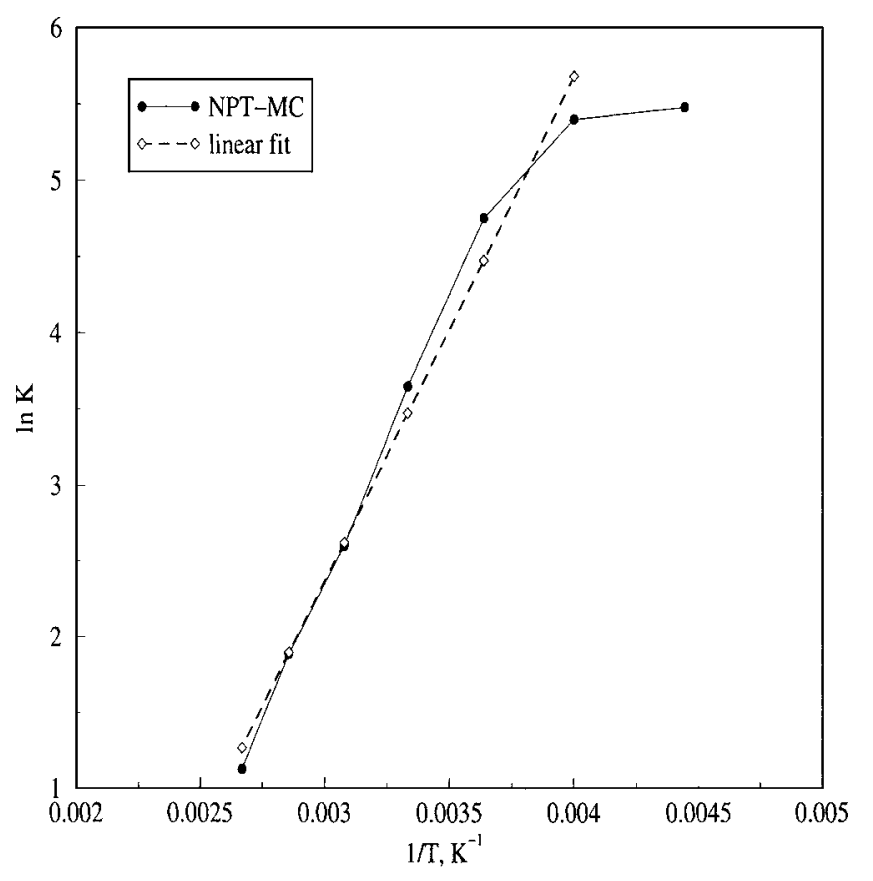

FIG. 13. van't Hoff plot of two orientations in the crystals of 4-vinyl benzoic acid. $K$ is the ratio of the population of major and minor conformers.

peratures 100,350 , and $375 \mathrm{~K}$ and at 1 atm pressure. The view is along the crystallographic $a$ axis. The crystal structure at $100 \mathrm{~K}$ is highly ordered. At this temperature, the molecules exhibit low-amplitude translational, rotational, and torsional motion. The structure at $350 \mathrm{~K}$ shows considerable disorder associated with translational, rotational, and torsional degrees of freedom. The structure at $375 \mathrm{~K}$ shows a reasonably larger translational, orientational, and conformational disorder and at this temperature molecules exhibit large-amplitude translational, rotational, and torsional motion. In this light, it is easier to understand the complete disappearance of peaks in various atom-atom rdfs at higher temperatures as shown in Fig. 7.

Figure 13 shows the van't Hoff plot of two orientations in the 4-vinyl benzoic acid crystal. It is a plot of $\ln K$ vs $1 / T$, where $K$ is the equilibrium constant between two orientations with torsional angles $0^{\circ}$ and $180^{\circ}$ and $K$ is given by the equation

$$
K=\frac{\text { population of major conformer }}{\text { population of minor conformer }} .
$$

The nonlinear behavior at lower temperatures in the van't Hoff plot explains that the difference of enthalpy $(\Delta H)$ between two conformers is not constant over all the temperature range. A linear fit has been fitted to the van't Hoff plot excluding the point corresponding to lower temperatures, and from the slope (see Table III) the $\Delta H$ has been found to be $27.5 \mathrm{~kJ} / \mathrm{mol}$. Recently, nonlinear behavior in the van't Hoff plot of major and minor orientations also has been observed in the case of stilbene and azobenzene ${ }^{35}$ and in the case of $\mathrm{N}$-(4-methylbenzylidene)-4-methylaniline. ${ }^{36}$ Figure 14 shows the Arrhenius plot of $k$ against reciprocal temperature. Here, $k$ is the rate of conversion between major and minor conformers. It is calculated from the number of jumps
TABLE III. The slope and constants $(Y=M X+C)$ of the van't Hoff plot and the enthalpy difference for the two conformers. Note: $\Delta H=\left(-k_{B}\right)$ (slope).

\begin{tabular}{cc}
\hline \hline & NPT-MC \\
\hline Slope & 3311.15 \\
Constant & -7.56473 \\
$\Delta H(\mathrm{~kJ} / \mathrm{mole})$ & 27.5 \\
\hline \hline
\end{tabular}

of molecules from $|\phi|<90^{\circ}$ to $|\phi| \geqslant 90^{\circ}$. A linear fit for the plot excluding the points corresponding to low temperatures gives an activation energy for interconversion as $27.38 \mathrm{~kJ} / \mathrm{mol}$.

In Fig. 15 the temperature dependence of the unit-cell volume and total interaction energy for both $\mathrm{FB}$ and $\mathrm{RB}$ calculations are shown. At low temperatures, both FB and $\mathrm{RB}$ calculations result in comparable unit-cell volume and lattice energy. This is because the disorder is negligible at low temperatures. But at higher temperatures, the deviation in unit-cell volume and energy between FB and RB calculations is considerably large as the conformational disorder increases by a larger extent. The unit-cell volume increases by $5.25 \%$ in the case of FB calculations at $375 \mathrm{~K}$ when compared to $\mathrm{RB}$ calculations. The total interaction energy increases by $7.86 \%$ in the case of $\mathrm{FB}$ calculations at $375 \mathrm{~K}$ when compared to RB calculations. This excess amount in unit-cell volume and total interaction energy is merely due to the conformational disorder associated with the flip-flop motion of vinyl groups. Also, at higher temperatures the RB calculations underestimate the experimentally calculated unit-cell volume, whereas the FB calculations reproduce it reasonably well. This demonstrates the importance of including the flexibility in the simulations for molecules with considerable flexibility to reproduce the structural quantities correctly.

\section{Bond-length variation and disorder}

The results from constrained optimizations of molecular structures differing in torsional angle $(\phi)$ and the temperature dependence of torsional angle distributions from simulations are combined to understand the temperature dependence of vinyl $\mathrm{C}=\mathrm{C}$ and $\mathrm{C}-\mathrm{Ph}$ bond lengths. The dependence of the vinyl $\mathrm{C}=\mathrm{C}$ and $\mathrm{C}-\mathrm{Ph}$ bond lengths on the torsional angle was first computed for a single molecule of 4-vinyl benzoic acid by doing constrained optimizations for molecular structures in varying torsional angle $\phi$. These results are shown in Figs. 16(a) and 16(b). From the Monte Carlo (MC) simulations, we obtain the torsional angle for each of the 240 molecules of 4-vinyl benzoic acid in the crystal at each MC step. We then obtain the corresponding $\mathrm{C}=\mathrm{C}$ and $\mathrm{C}-\mathrm{Ph}$ bond lengths from the $a b$ initio derived curves [Figs. 16(a) and 16(b)] for each molecule. An average over all these bond lengths for $N$ molecules and all MC steps yielded the average $\mathrm{C}=\mathrm{C}$ and $\mathrm{C}-\mathrm{Ph}$ bond lengths of 4-vinyl benzoic acid at a given temperature. This averaging has been carried out for the torsional angle distributions at varying temperatures. These results are shown in Figs. 16(c) and 16(d). From the figures it is clearly seen that the $\mathrm{C}=\mathrm{C}$ bond length decreases and $\mathrm{C}-\mathrm{Ph}$ bond length increases as a func- 


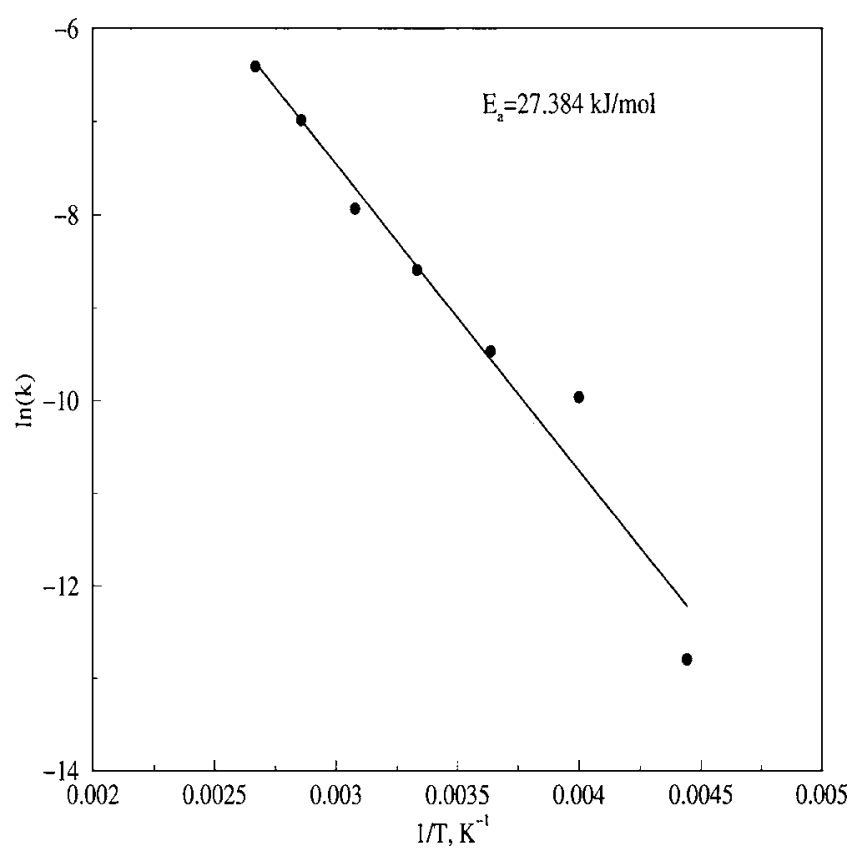

FIG. 14. Arrhenius plot of $k$ against reciprocal temperature. $k$ is the rate of conversion from major to minor conformers.

tion of temperature. Even though in magnitude the variation for the $\mathrm{C}=\mathrm{C}$ bond length observed in the experiments is high [i.e., $\Delta d=0.037 \AA$ for the temperature range 108-293 K (Ref. 14)], the present calculations predict the trend qualitatively very well. Also, it is to be noted that the bond-length variations of $\mathrm{C}=\mathrm{C}$ and $\mathrm{C}-\mathrm{Ph}$ are not very significant in the temperature range of $100-300 \mathrm{~K}$, where the disorder is only less than $3 \%$. Above $300 \mathrm{~K}$, both these bond lengths show larger variation in magnitude. As the tempera-

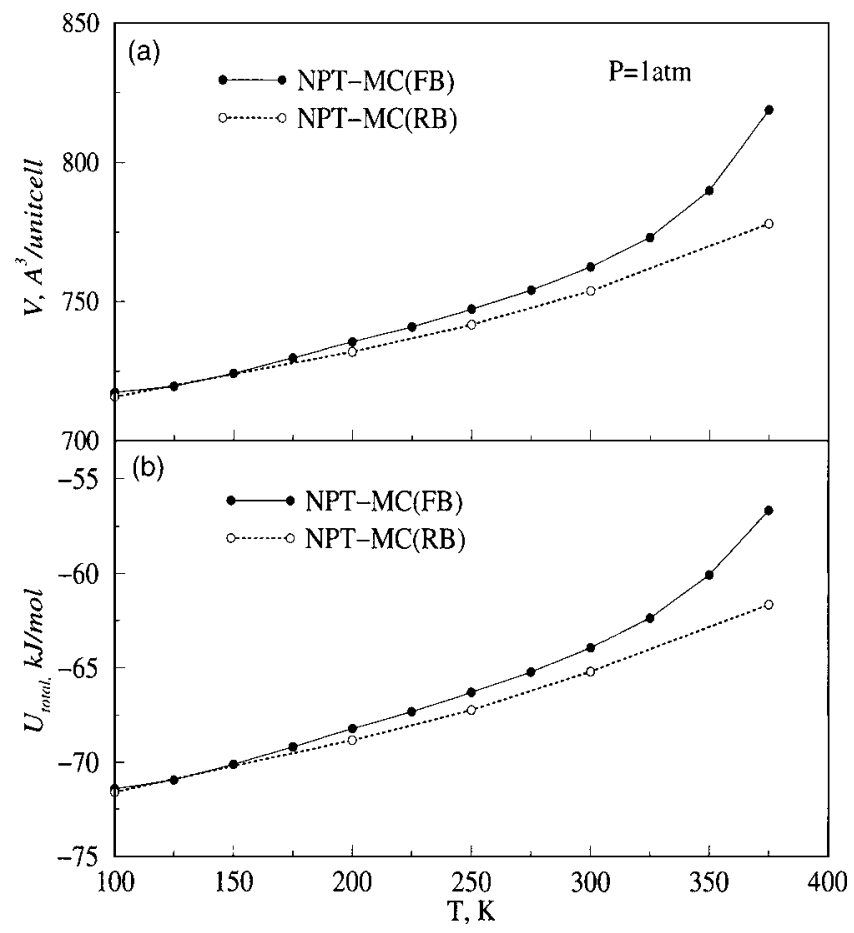

FIG. 15. Temperature dependence of (a) unit-cell volume and (b) total interaction energy for both flexible-body and rigid-body calculations.
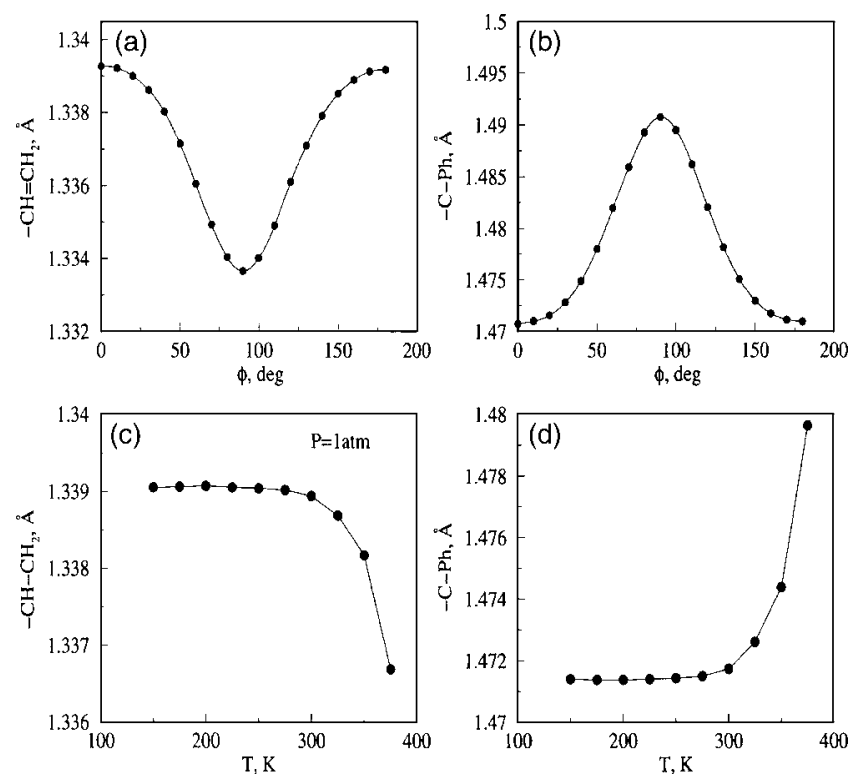

FIG. 16. (a) Variation of vinyl $\mathrm{C}=\mathrm{C}$ bond length with the torsional angle, derived from ab initio calculations. (b) Variation of $\mathrm{C}-\mathrm{Ph}$ bond length with the torsional angle, derived from ab initio calculations. (c) Variation of vinyl $\mathrm{C}=\mathrm{C}$ bond length as a function of temperature. (d) Variation of $\mathrm{C}-\mathrm{Ph}$ bond length as a function of temperature.

ture dependence of conformational disorder is described reasonably well from these simulations, the reason for the incorrect magnitude of the calculated bond lengths arises from the inaccurate torsional angle dependence of these bond lengths calculated from $a b$ initio calculations. More accurate $a b$ initio electronic calculation methods ${ }^{19}$ such as CI, MP2, MP3, and MP4 ${ }^{19}$ can be carried out to get more accurate torsional angle dependence of $\mathrm{C}=\mathrm{C}$ and $\mathrm{C}-\mathrm{Ph}$ bond lengths. In that case, the temperature dependence of these bond lengths may reproduce experimental results more correctly.

\section{CONCLUSIONS}

The molecular simulations at different temperatures prove the dynamic nature of the intramolecular orientational disorder associated with the flip-flop motion of vinyl groups in 4-vinyl benzoic acid crystal. The simulations could reproduce the structure of the crystal over the temperature range of $100-375 \mathrm{~K}$ reasonably well. The calculations could quantify the percentage of minor conformers to be less than $3 \%$ below $300 \mathrm{~K}$, which is consistent with experimental observations. ${ }^{14}$ The percentage of disorder at $350 \mathrm{~K}$ is $13.17 \%$, which again can be compared to $11.8 \%$ of minor conformers observed at $363 \mathrm{~K}$ experimentally. ${ }^{14}$

The van't Hoff plot of two orientations showed the nonlinear behavior at low temperatures, explaining that the difference in enthalpy for two conformers is not constant over all the temperatures. This type of behavior in the van't Hoff plot has also been reported in the recent works by Ogawa and co-workers in the case of stilbene and azobenzene ${ }^{35}$ and in the case of $\mathrm{N}$-(4-methylbenzylidene)-4-methylaniline. ${ }^{36}$

The anomalous shrinkage of $\mathrm{C}=\mathrm{C}$ vinyl bond length as a function of temperature has been explained using a set of constrained optimizations and the temperature dependence of 
torsional angle distribution data from simulations. It has also been observed that the $\mathrm{C}-\mathrm{Ph}$ bond length increases as a function of temperature.

\section{ACKNOWLEDGMENTS}

The author wishes to thank Professor S. Yashonath for helpful discussions and encouragement and JNCASR for financial support. Also, the author wishes to thank Dr. Karthikeyan (RRI), Sridhar, Martha, and Ananda Ganesh for their encouragement.

${ }^{1}$ N. F. Mott, Philos. Mag. 13, 93 (1966).

${ }^{2}$ P. W. Anderson, B. C. Halperin, and C. M. Varma, Philos. Mag. 25, 1 (1972).

${ }^{3}$ V. P. Kolesov, Thermochim. Acta 266, 129 (1995).

${ }^{4}$ D. L. Price, M. L. Saboungi, and F. J. Bermejo, Rep. Prog. Phys. 66, 407 (2003).

${ }^{5}$ J. P. Amoureux, M. Bee, and J. C. Damien, Acta Crystallogr., Sect. B: Struct. Crystallogr. Cryst. Chem. 36, 2633 (1980).

${ }^{6}$ J. P. Amoureux and M. Foulon, Acta Crystallogr., Sect. B: Struct. Sci. 43, 470 (1987).

${ }^{7}$ S. R. Salman, E. Z. Said, and K. F. Abas, Thermochim. Acta 111, 21 (1987).

${ }^{8}$ J. N. Sherwood, The Plastically Crystalline State (Wiley, New York, 1979).

${ }^{9}$ A. Katrusiak, Acta Crystallogr., Sect. B: Struct. Sci. 56, 872 (2000).

${ }^{10}$ K. Ogawa, T. Sano, S. Yoshimura, Y. Takeuchi, and K. Toriumi, J. Am. Chem. Soc. 114, 1041 (1992).

${ }^{11}$ J. Harada and K. Ogawa, J. Am. Chem. Soc. 123, 10884 (2001).

${ }^{12}$ J. Bernstein and K. Mirsky, Acta Crystallogr., Sect. A: Cryst. Phys., Diffr., Theor. Gen. Crystallogr. 34, 161 (1978).

${ }^{13}$ K. Ogawa, J. Harada, and S. Tomoda, Acta Crystallogr., Sect. B: Struct.
Sci. 51, 240 (1995).

${ }^{14}$ N. Yasuda, H. Uekusa, and Y. Ohashi, J. Mol. Struct. 647, 217 (2003).

${ }^{15}$ N. Yasuda, H. Uekusa, and Y. Ohashi, Acta Crystallogr., Sect. C: Cryst. Struct. Commun. 56, 1364 (2000).

${ }^{16}$ R. Car and M. Parrinello, Phys. Rev. Lett. 55, 2471 (1985).

${ }^{17}$ M. J. Frisch, G. W. Trucks, H. B. Schlegel et al., GAUSSIAN 98, revision A.9, Gaussian, Inc., Pittsburgh, PA, 1998.

${ }^{18}$ G. Filippini and A. Gavezzotti, Acta Crystallogr., Sect. B: Struct. Sci. 49, 868 (1993).

${ }^{19}$ S.-N. Luo and T. J. Ahrens, Appl. Phys. Lett. 82, 1836 (2003).

${ }^{20}$ K. Lu and Y. Li, Phys. Rev. Lett. 80, 4474 (1998).

${ }^{21}$ G. F. Velardez, S. Alavi, and D. L. Thompson, J. Chem. Phys. 119, 6698 (2003).

${ }^{22}$ M. Sprik, U. Rothlisberger, and M. L. Klein, J. Phys. Chem. B 101, 2745 (1997).

${ }^{23}$ D. W. Grieg and G. S. Pawley, Mol. Phys. 89, 677 (1996).

${ }^{24}$ A. Hehre, E. Radom, A. Schleyer, and J. A. Pople, Ab Initio Molecular Orbital Theory (Wiley-Interscience, New York, 1986).

${ }^{25}$ M. Parrinello and A. Rahman, Phys. Rev. Lett. 45, 1196 (1980).

${ }^{26}$ N. Metropolis, A. W. Rosenbluth, M. N. Rosenbluth, A. H. Teller, and E. Teller, J. Chem. Phys. 21, 1087 (1953).

${ }^{27}$ S. Yashonath and C. N. R. Rao, Chem. Phys. Lett. 119, 22 (1985).

${ }^{28}$ N. A. Murugan and S. Yashonath, J. Phys. Chem. B 108, 17403 (2004).

${ }^{29}$ N. A. Murugan, P. C. Jha, S. Yashonath, and S. Ramasesha, J. Phys. Chem. B 108, 4178 (2004).

${ }^{30}$ N. A. Murugan and S. Yashonath, J. Phys. Chem. B 109, 1433 (2005).

${ }^{31}$ S. Yashonath and C. N. R. Rao, J. Phys. Chem. B 90, 2552 (1980).

${ }^{32}$ N. A. Murugan and S. Yashonath, J. Phys. Chem. B 109, 2014 (2005).

${ }^{33}$ D. J. Bounds, M. L. Klein, and G. N. Patey, J. Chem. Phys. 72, 5348 (1980).

${ }^{34}$ S. Nose and M. L. Klein, J. Chem. Phys. 78, 6928 (1983).

${ }^{35}$ J. Harada and K. Ogawa, J. Am. Chem. Soc. 126, 3539 (2004).

${ }^{36}$ J. Harada, M. Harakawa, and K. Ogawa, Acta Crystallogr., Sect. B: Struct. Sci. 60, 589 (2004). 